\title{
Sonoanatomical Change of Phrenic Nerve According to Posture During Ultrasound-Guided Stellate Ganglion Block
}

\author{
Eui Soo Joeng, MD, Young Cheol Jeong, MD, Bum Jun Park, MD, Seok Kang, MD, \\ Seung Nam Yang, MD, PhD, Joon Shik Yoon, MD, PhD \\ Department of Physical Medicine and Rehabilitation, Korea University Guro Hospital, Seoul, Korea
}

Objective To evaluate the risk of phrenic nerve injury during ultrasound-guided stellate ganglion block (US-SGB) according to sonoanatomy of the phrenic nerve, and determine a safer posture for needle insertion by assessing its relationship with surrounding structure according to positional change.

Methods Twenty-nine healthy volunteers were recruited and underwent ultrasound in two postures, i.e., supine position with the neck extension and head rotation, and lateral decubitus position. The transducer was placed at the anterior tubercle of the C6 level to identify phrenic nerve. The cross-sectional area (CSA), depth from skin, distance between phrenic nerve and anterior tubercle of C6 transverse process, and the angle formed by anterior tubercle, posterior tubercle and phrenic nerve were measured.

Results The phrenic nerve was clearly identified in the intermuscular fascia layer between the anterior scalene and sternocleidomastoid muscles. The distance between the phrenic nerve and anterior tubercle was $10.33 \pm 3.20$ $\mathrm{mm}$ with the supine position and $9.20 \pm 3.31 \mathrm{~mm}$ with the lateral decubitus position, respectively. The mean CSA and skin depth of phrenic nerve were not statistically different between the two positions. The angle with the supine position was $48.37^{\circ} \pm 27.43^{\circ}$, and $58.89^{\circ} \pm 30.02^{\circ}$ with the lateral decubitus position. The difference of angle between the two positions was statistically significant.

Conclusion Ultrasound is a useful tool for assessing the phrenic nerve and its anatomical relation with other cervical structures. In addition, lateral decubitus position seems to be safer by providing wider angle for needle insertion than the supine position in US-SGB.

Keywords Stellate ganglion, Phrenic nerve, Ultrasound, Posture

Received May 6, 2015; Accepted August 12, 2015

Corresponding author: Joon Shik Yoon

Department of Physical Medicine and Rehabilitation, Korea University Guro Hospital, 148 Gurodong-ro, Guro-gu, Seoul 08308, Korea Tel: +82-2-2626-1500, Fax: +82-2-2626-1513, E-mail: rehab46@korea.ac.kr

() This is an open-access article distributed under the terms of the Creative Commons Attribution Non-Commercial License (http://creativecommons.org/ licenses/by-nc/4.0) which permits unrestricted noncommercial use, distribution, and reproduction in any medium, provided the original work is properly cited. Copyright $\odot 2016$ by Korean Academy of Rehabilitation Medicine 


\section{INTRODUCTION}

Stellate ganglion block (SGB) is a common intervention in the diagnosis and management of sympathetically mediated pain and vascular insufficiency of the upper extremities. In addition, this type of anesthetic block is commonly utilized for the treatment of pain conditions of the head and neck regions as well as the upper limbs, such as phantom pain, postherpetic neuralgia, cancer pain, cardiac arrhythmias, orofacial pain, and vascular headache [1].

The stellate ganglion lies medial to the scalene muscles, lateral to the longus colli muscle, anterior to the transverse processes and prevertebral fascia, and superior to the subclavian artery and the posterior aspect of the pleura.

The SGB is typically performed at the C6 level. At C6 level, the cervical sympathetic chain is anterior to the longus colli muscle, embedded within the prevertebral fascia. There are many important soft-tissue structures that can be unintentionally injured at these levels during needle placement for SGB, including the trachea, thyroid gland, esophagus, arteries (carotid, vertebral, inferior thyroidal, deep and ascending cervical), cervical nerve roots, and external jugular vein. In addition, small nerves such as phrenic nerve or spinal accessory nerve should be identified to avoid inadvertent injury.

The phrenic nerve arises from the ventral ramus of the fourth cervical nerve with contributions from the third and fifth cervical nerves [2]. The nerve lies on the anterior surface of the anterior scalene muscle behind its fascia at vertebral level C6, and runs distally following the upper portion of the anterior scalene muscle. It is mostly made up of motor fibers that regulate diaphragmatic contraction. In addition, it provides the pleura and many components of the mediastinum and upper abdomen with sensory innervation [3]. The phrenic nerve could be located on the needle pathway when performing SGB. Diaphragmatic paralysis due to concomitant phrenic nerve block could occur, hence, it should be prevented especially in patients with chronic respiratory disease, chronic obstructive pulmonary disease (COPD), bronchial asthma, or high body mass index (BMI) [2]. Thus, although the ultrasound provides excellent real-time visualization of the neurovascular structures, the identification of phrenic nerve and assessment of its location during SGB is important to avoid the needle-induced injury.

Many detailed studies address the risk of injury to the esophagus, trachea, or major vessels when performing SGB, however, descriptions of the phrenic nerve are less common, and to our best knowledge, there are no prior reports on the sonoanatomy of phrenic nerve during SGB. The aim of this study was to evaluate the risk of phrenic nerve injury during ultrasound-guided SGB (USSGB) by investigating the sonoanatomy of the phrenic nerve in the cervical region. In addition, we determined the safer position for SGB by examining the variations of the phrenic nerve and its relationship with surrounding structure according to the change of position.

\section{MATERIALS AND MEHTODS}

The protocol for this prospective study was approved by the Institutional Review Board of the Korea University Hospital. Informed consent was obtained from all patients prior to each US examination.
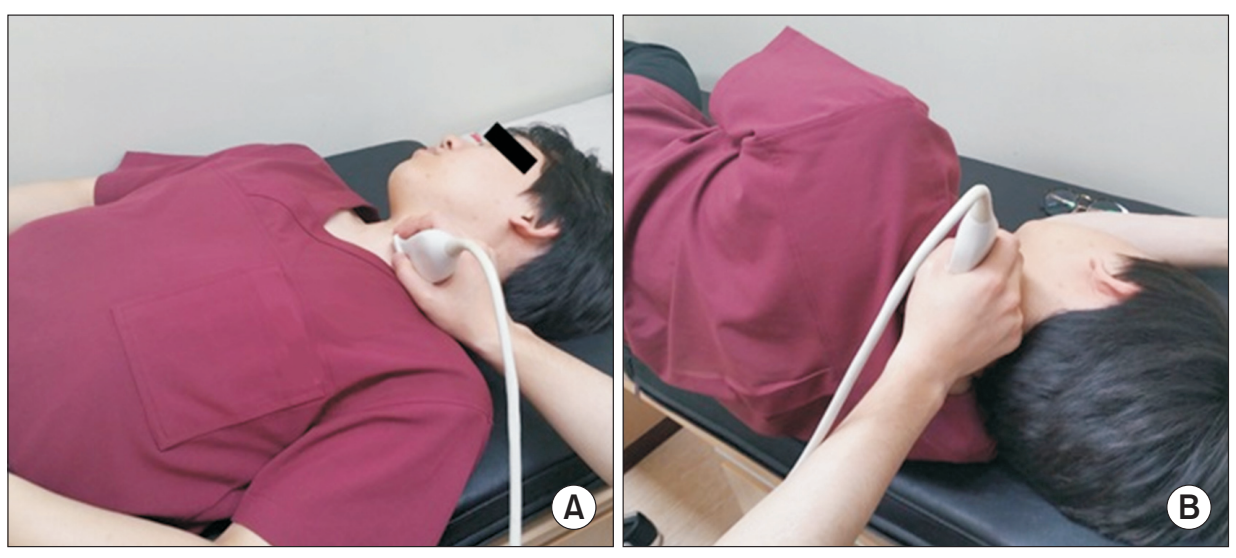

Fig. 1. Sonographic evaluations were performed in two postures. (A) Supine position with the neck slightly extended, and the head rotated slightly to the opposite side to be blocked. (B) Lateral decubitus position with the side to be treated uppermost. 


\section{Subjects}

A total of 29 healthy volunteers were recruited for this study. There were 18 males and 11 females, with an overall mean age $45.9 \pm 19.4$ years (range, $18-81$ years). We excluded those who met the following exclusion criteria: 1) age $<18$ years, 2) history of cervical disorder, 3 ) history of cervical trauma or surgery, and 4) peripheral neuropathy.

\section{Sonographic technique and posture of patient}

All sonographic examinations were performed using a Philips HD15 equipped with a linear probe. US was performed with the volunteers in two postures, i.e., 1) supine position with the neck slightly extended, and the head rotated slightly to the opposite side to be blocked [2-5] $[3,4]$, and 2) lateral decubitus position with the side to be treated uppermost [4-6] (Fig. 1).

The transverse processes were initially examined in the axial plane for assessment of the level of the cervical vertebra. The transducer was initially placed at the level of the cricoid cartilage, and traced caudally to identify the typical appearance of the $\mathrm{C} 6$ transverse process, with a prominent anterior tubercle, a smaller posterior tubercle, and the $\mathrm{C} 6$ nerve root. The level was confirmed by scanning caudally to visualize the $\mathrm{C} 7$ transverse process. The anterior tubercle of the transverse process is selectively absent in the $\mathrm{C} 7$ vertebra and considered as the key landmark of $\mathrm{C} 7$ vertebra.

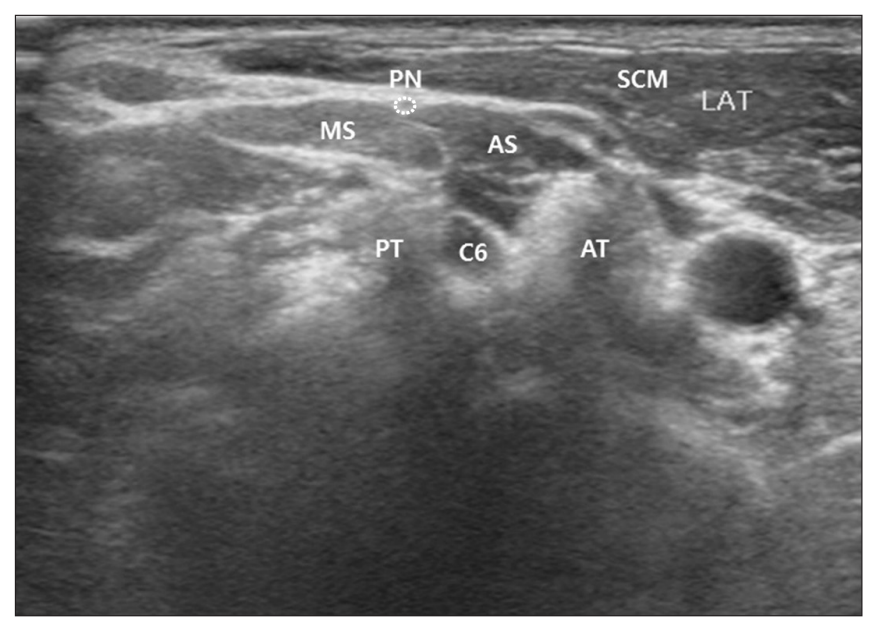

Fig. 2. Sonographic landmarks, anterior scalene muscle (AS), middle scalene muscle (MS), sternocleidomastoid muscle (SCM), anterior tubercle (AT), and phrenic nerve (PN), were identified on the plane of vertebral C6 level. The margin of phrenic nerve was presented as dotted line. C6, C6 nerve root; PT, posterior tubercle.
The phrenic nerve was observed by first assessing the anterior scalene muscle, which was seen immediately behind the sternocleidomastoid muscle, lateral to the internal jugular vein and medial to the brachial plexus roots. The nerve appeared as an ovoid hypoechogenic structure, located on the anterior surface of the anterior scalene muscle behind its fascia at vertebral level C6.

Sonographic landmarks, anterior scalene muscle, middle scalene muscle, sternocleidomastoid muscle, anterior tubercle, and phrenic nerve, were identified on the plane of vertebral C6 level (Fig. 2).

Depth of phrenic nerve from skin and distance between phrenic nerve and anterior tubercle of C6 transverse process

We measured the shortest distance between the epineurium of phrenic nerve and the surface of skin. We also measured the shortest distance between the epineurium of phrenic nerve and the anterior tubercle of the C6 transverse process (Fig. 3).

\section{The angle which is formed by anterior tubercle, posterior} tubercle and phrenic nerve

An imaginary line $(\overline{\mathrm{AB}})$ was drawn passing the upper

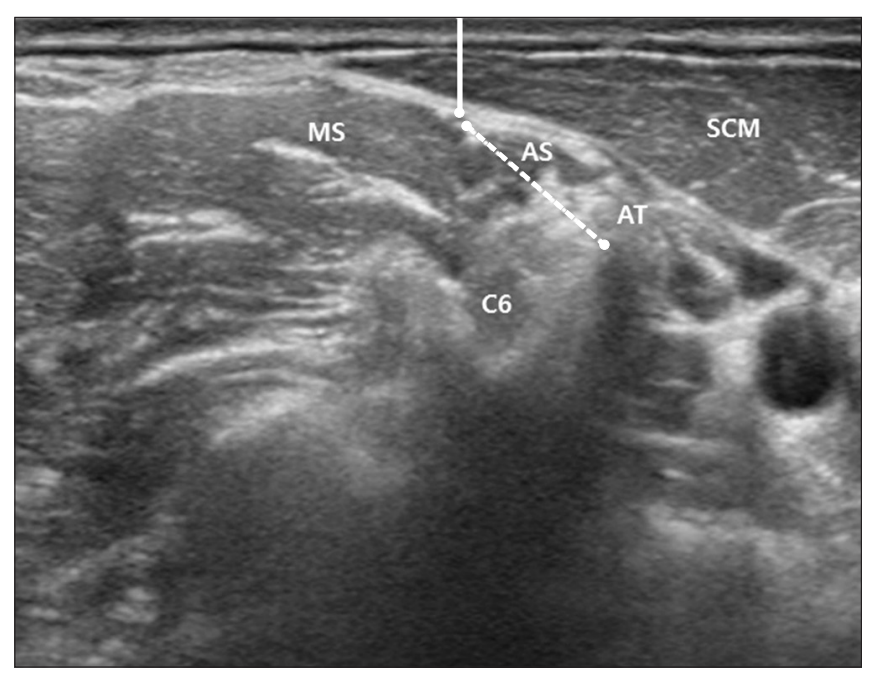

Fig. 3. Depth of phrenic nerve from skin was measured by the shortest distance between the epineurium of phrenic nerve and the surface of skin (presented as solid line). The shortest distance between the epineurium of phrenic nerve and the anterior tubercle (AT) of the C6 transverse process was also measured (presented as dotted line). SCM, sternocleidomastoid muscle; AS, anterior scalene muscle; MS, middle scalene muscle; $\mathrm{C} 6$, C6 nerve root. 
margin of posterior tubercle ('A') and anterior tubercle ('B'). In addition, another imaginary line $(\overline{\mathrm{CD}})$ was drawn passing the two points both the lower margin of epineurium of phrenic nerve ('C') and the upper margin of anterior tubercle (' $\mathrm{D}$ '). The two imaginary lines were extended above and the point of intersection was designated as 'E. The angle formed by the lower margin of epineurium of phrenic nerve ('C'), point ' $E$ ', and the upper margin of posterior tubercle ('A') ( $\angle$ CEA) was measured (Fig. 4).

The cervical sympathetic chain, the target point of SGB (represented as shaded rectangle on Fig. 5), lies anterior to the longus colli muscle, and is embedded within the prevertebral fascia. When performing SGB, the anterior tubercle blocks the needle from reaching the target point, and the phrenic nerve is located in the needle pathway. Therefore, the angle formed by posterior tubercle, anterior tubercle and phrenic nerve, is a safe angle to perform successful SGB without phrenic nerve injury.

\section{Statistical analysis}

Statistical analyses were performed using the SPSS software package (ver. 20.0). A normal distribution was confirmed by Kolmogorov-Smirnov test. Paired t-test was used for comparing the depth of phrenic nerve from skin,
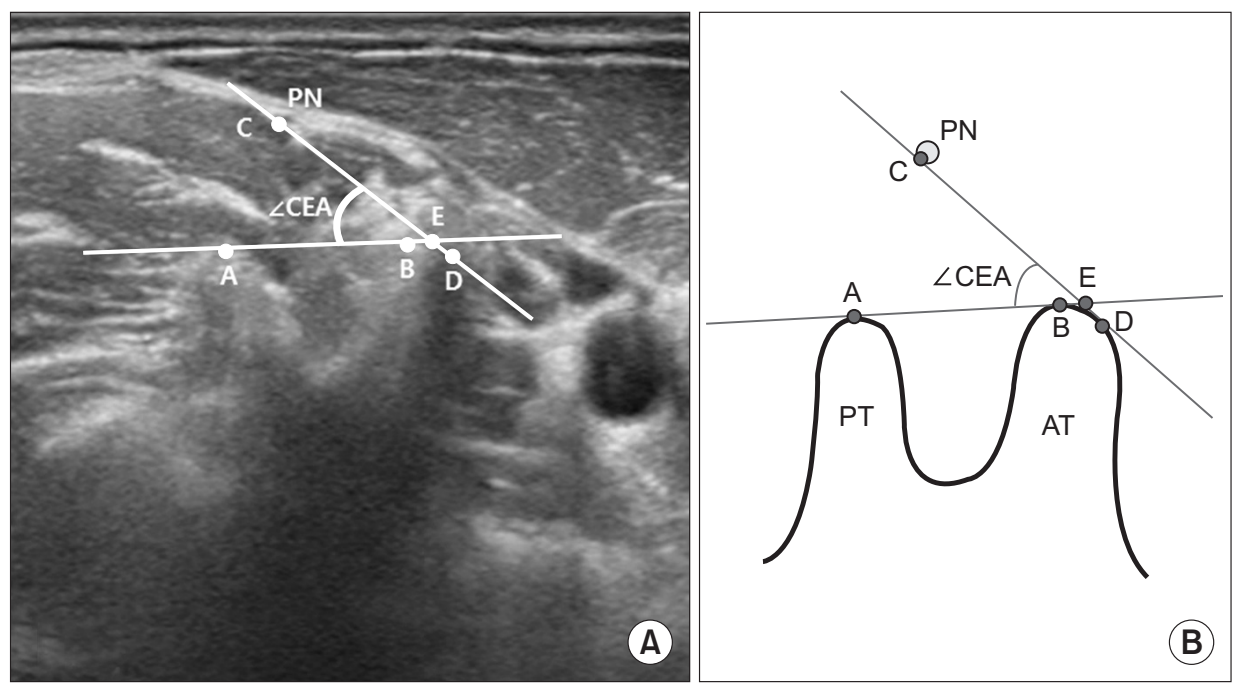

Fig. 4. The angle formed by the lower margin of epineurium of $\mathrm{PN}$ (point ' $C$ '), point ' $E$ ', and the upper margin of PT (point ' $\mathrm{A}$ ') ( $\angle \mathrm{CEA})$ was described on ultrasound image (A) and schematic image (B). 'A', upper margin of PT; 'B', point on the AT created by connecting PT and AT; ' $\mathrm{C}$ ', lower margin of epineurium of $\mathrm{PN}$; ' $\mathrm{D}$ ', point on the AT created by connecting lower margin of PN and AT; 'E', crossed point of extended line $\mathrm{AB}$ and CD; PN, phrenic nerve; PT, posterior tubercle; AT, anterior tubercle.
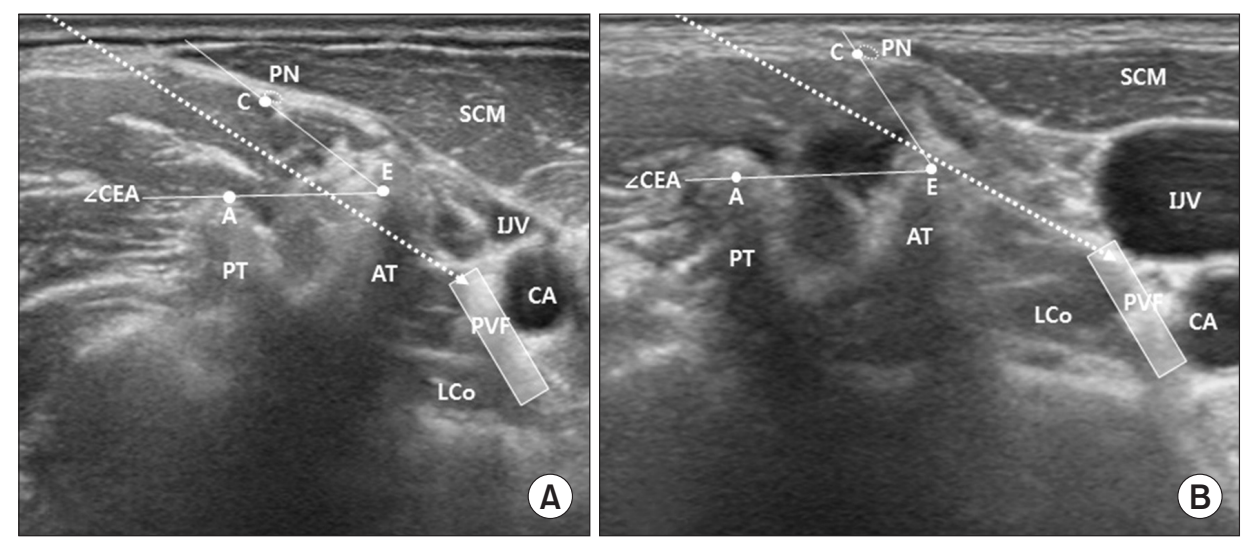

Fig. 5. Virtual needle pathways were described as dotted arrows. The target point was represented as shaded rectangle. (A) When the safe angle ( $\angle \mathrm{CEA})$ is small, if the needle aims at the target point directly, the needle would be blocked by AT and cannot reach the target point. (B) When the needle pathway is positioned within the range of safe angle ( $\angle \mathrm{CEA})$, the needle could reach the target point without contacting the AT and PN. Larger safe angle $(\angle \mathrm{CEA})$ provides greater possibility to approach the target point safely. 'A', upper margin of $\mathrm{PT}$; ' $\mathrm{C}$ ', lower margin of epineurium of $\mathrm{PN}$; ' $\mathrm{E}$ ', crossed point of extended line $\mathrm{AB}$ and $\mathrm{CD}$; AT, anterior tubercle; PN, phrenic nerve; PT, posterior tubercle; IJV, internal jugular vein; CA, carotid artery; PVF, prevertebral fascia; LCo, longus colli muscle. 
distance between the phrenic nerve and anterior tubercle of the transverse process, and the angle that is formed by anterior tubercle, posterior tubercle and phrenic nerve ( $\angle$ CEA) between supine and lateral decubitus positions. Wilcoxon signed-rank test was used for comparing the CSA of phrenic nerve between supine and lateral decubitus positions. Correlation analyses were performed for evaluation of correlation between the variants.

\section{RESULTS}

Eighteen male and 11 female volunteers were enrolled. The characteristics of volunteers were summarized in Table 1 . The mean age of 29 volunteers was $45.9 \pm 19.4$ years (range, $18-81$ years). The mean BMI was $23.1 \pm 3.1 \mathrm{~kg} / \mathrm{m}^{2}$ (range, $17.6-31.2 \mathrm{~kg} / \mathrm{m}^{2}$ ).

The sonographic values of the phrenic nerve were shown in Table 2. The mean CSA of phrenic nerve was $0.68 \pm 0.35 \mathrm{~mm}^{2}$ in supine position, and $0.66 \pm 0.35 \mathrm{~mm}^{2}$ in lateral decubitus position. The depth of phrenic nerve from skin was $5.82 \pm 2.62 \mathrm{~mm}$ in supine position, and $6.04 \pm 2.15 \mathrm{~mm}$ in lateral decubitus position. The mean

Table 1. Clinical characteristics of volunteers

\begin{tabular}{lc}
\hline Parameter & Value \\
\hline Sex $($ male:female $)$ & $18: 11$ \\
Age $(\mathrm{yr})$ & $45.9 \pm 19.4(18-81)$ \\
\hline Height $(\mathrm{cm})$ & $169.9 \pm 8.1(150-183)$ \\
Weight $(\mathrm{kg})$ & $67.0 \pm 12.0(48-99)$ \\
\hline BMI $\left(\mathrm{kg} / \mathrm{m}^{2}\right)$ & $23.1 \pm 3.1(17.6-31.2)$ \\
\hline
\end{tabular}

Values are presented as mean \pm standard deviation (range) or number.
CSA of phrenic nerve and depth of phrenic nerve from skin was not different between the two positions. The distance between the phrenic nerve and anterior tubercle in supine position was $10.33 \pm 3.20 \mathrm{~mm}$ and $9.20 \pm 3.31$ $\mathrm{mm}$ in lateral decubitus position, respectively. Though the distance tended to decrease in the lateral decubitus position, it was not statistically significant. Twenty-two of 29 volunteers showed increment of the angle $(\angle \mathrm{CEA})$ with the position change from supine to lateral decubitus position, but 7 showed decrement. The mean angle ( $\angle \mathrm{CEA}$ ) was $48.37^{\circ} \pm 27.43^{\circ}$ with the supine position, and $58.89^{\circ} \pm 30.02^{\circ}$ with the lateral decubitus position. The angle was significantly wider in lateral decubitus position.

BMI was significantly correlated with the depth of phrenic nerve in both positions ( $\mathrm{p}=0.022, \mathrm{r}=0.455$ in supine position; $\mathrm{p}<0.001, \mathrm{r}=0.656$ in lateral decubitus position). In addition, BMI was significantly correlated with the angle ( $\angle \mathrm{CEA})$ in supine position $(\mathrm{p}=0.001, \mathrm{r}=0.625)$, while no significant correlation was found in lateral decubitus position ( $\mathrm{p}=0.061, \mathrm{r}=0.380$ ). No significant correlations were found between the characteristics of subjects and the changed values of sonographic parameters.

\section{DISCUSSION}

In performing cervical procedures, several anatomic structures have to be considered to avoid serious complications. The posterior cervical triangle includes a number of vascular structures, lymph nodes and components of the cervical and brachial plexus [7]. Severe complications are reported, including immediate seizures, hematoma formation with airway compromise, and death [8]. Spread of local anesthetic into the epidural space

Table 2. Sonographic values of phrenic nerve

\begin{tabular}{lccc}
\hline & Supine & Lateral decubitus & p-value \\
\hline CSA $\left.\left(\mathrm{mm}^{2}\right)^{\mathrm{a}}\right)$ & $0.68 \pm 0.35$ & $0.66 \pm 0.35$ & 0.279 \\
& $(0.60,0.50-0.75)$ & $(0.60,0.50-0.75)$ & \\
Skin depth $\left.(\mathrm{mm})^{\mathrm{b}}\right)$ & $5.82 \pm 2.62$ & $6.04 \pm 2.15$ & 0.552 \\
Distance from anterior tubercle $(\mathrm{mm})^{\mathrm{b})}$ & $10.33 \pm 3.20$ & $9.20 \pm 3.31$ & 0.085 \\
$\angle \mathrm{CEA}\left({ }^{\mathrm{b}}\right)^{\mathrm{b}}$ & $48.37 \pm 27.43$ & $58.89 \pm 30.02$ & $0.014^{*}$ \\
\hline
\end{tabular}

Values are presented as mean \pm standard deviation (median, interquartile range).

CSA, cross-sectional area; CEA, the angle formed by the lower margin of epineurium of phrenic nerve ('C'), point ' $E$ ', and the upper margin of posterior tubercle ('A').

${ }^{\text {a) }} \mathrm{CSA}$ was analyzed by Wilcoxon signed-rank test.

${ }^{\mathrm{b})}$ Paired t-test was used for comparing the values with two positions.

${ }^{*} \mathrm{p}<0.05$. 
can result in hypotension and bradycardia. Unintentional esophageal puncture and pneumothorax are also reported as severe complications. Associated brachial plexus block is reported in up to $10 \%$ of patients. The recurrent laryngeal nerve may also be blocked, resulting in a hoarse voice and unilateral vocal cord paresis [9]. Some case reports of development of Horner syndrome cite bilateral occurrence, which may cause visual impairment [10].

The phrenic nerve could be placed in the needle-path and partial phrenic blocks could occur when performing various anesthetic procedures of cervical area. An accidental diaphragmatic paresis secondary to the cervical procedure is often asymptomatic but in cases of preexisting respiratory insufficiency, decompensation may occur rapidly post-procedure. It occurs in approximately $50 \%$ of cases, when performing supraclavicular brachial plexus block [11]. This side effect is possible even when diaphragmatic stimulation is not observed when performing the block. In addition to acute phrenic nerve block from local anesthesia, persistent phrenic nerve injury from interscalene block or internal jugular vein cannulation is reported [12]. Although US provides excellent direct and real-time visualization of the soft tissues of the neck [13], including neurovascular structures, the identification of phrenic nerve and assessment of its course before performing SGB is advisable to avoid intraprocedural needle-nerve contact .

Several studies on sonoanatomy of phrenic nerve are reported. In an anatomical study in cadavers and volunteers, Canella et al. [3] reported the mean diameter of the phrenic nerves of $0.6 \mathrm{~mm} \times 1.0 \mathrm{~mm}$ with no significant differences between the values according to the gender, age or examined side. Kessler et al. [2] also identified the phrenic nerve with US in both cadavers and volunteers. They measured the distance between the phrenic nerve and the ventral ramus of $\mathrm{C} 5$, and the depth of phrenic nerve from skin. The distance between the phrenic nerve and the ventral ramus of C5 was $1.8 \mathrm{~mm}$ at the level of cricoid cartilage, and the depth of phrenic nerve from skin was $5.4 \mathrm{~mm}$, respectively.

In our study, we measured the anatomical parameters of phrenic nerve at $\mathrm{C} 6$ level to evaluate the risk of phrenic nerve injury in SGB. The phrenic nerve was well demarcated by US in cervical area. In supine position, the CSA of phrenic nerve was $0.68 \pm 0.35 \mathrm{~mm}^{2}$ and the depth of phrenic nerve from the skin was $5.82 \pm 2.62 \mathrm{~mm}$. The distance between phrenic nerve and the anterior tubercle of C6 transverse process was $10.33 \pm 3.20 \mathrm{~mm}$ in supine position.

Two different needle approaches for US-SGB are reported. The first is the anterior paratracheal approach. The anterior paratracheal technique is performed as a standard technique to block stellate ganglion. However, this technique may result in a number of severe complications for e.g., subarachnoid or epidural injection, recurrent laryngeal nerve block, seizures, and blindness. The "in-plane" lateral approach is recently introduced [14]. The lateral approach seems to be an excellent alternative technique for avoiding the potential risks associated with the conventional approach.

We performed sonographic examinations for lateral approach with two different positions, i.e., supine and lateral decubitus. The general anatomical parameters of phrenic nerve, such as the CSA, depth of location and distance from the anterior tubercle of $\mathrm{C} 6$, were not significantly different according to the position. We measured the angle formed by anterior tubercle, posterior tubercle and phrenic nerve, to determine whether the intraprocedural location of the phrenic nerve is on the needle pathway. During SGB with lateral approach, because the anterior tubercle blocks the needle pathway, the needle should be positioned between the phrenic nerve and the anterior tubercle to avoid phrenic nerve injury. Thus, we regarded the angle formed by posterior tubercle, anterior tubercle and phrenic nerve as clinically important. After passing beyond the anterior tubercle without phrenic nerve injury, the needle tip could be advanced to the target point by regulating the direction of needle pathway. Therefore, larger angle $(\angle \mathrm{CEA})$ implies a greater possibility of approaching the target point safely.

In supine position, the angle was $48.37^{\circ} \pm 27.43^{\circ}$, while lateral decubitus position provides wider maneuvering space (the angle was $58.89^{\circ} \pm 30.02^{\circ}$ ) for the needle. The muscles and nerve are expected to move inferiorly due to the effect of gravity, because they are relatively softer and more movable than bony structures. Thus, lateral decubitus position may be safer to avoid injury of phrenic nerve and other related anatomical structures during SGB. However, although majority of subjects (22 of 29) showed increment of angle with lateral decubitus position, as compared to supine position, a small number of 
individuals showed decrement of angle with lateral decubitus position. Thus, lateral decubitus position could result in decrement of angle in few cases. Therefore, we recommend performing SGB in lateral decubitus position; however, checking both positions is necessary when it is difficult to reach the target point due to small angle in lateral decubitus position. To our knowledge, this is the first study to evaluate the risk of phrenic nerve injury during US-SGB according to anatomical relation between phrenic nerve and C6 transverse process, and the changes of values according to positions.

In addition, performing SGB with lateral approach in lateral decubitus position provides a more comfortable position for the physician, since the shoulder of the patient could disturb the injecting hand of the physician when performing SGB in supine position.

Our study had several limitations. The major limitation was that this was an observational study of neck sonoanatomy. The results of this study suggest only the potential for the reduction of risk of phrenic nerve injury during SGB in lateral decubitus position. The enhanced safety of performing SGB with lateral decubitus position can be evaluated only in a clinical trial. A randomized controlled trial comparing the incidence of complications due to phrenic nerve injury using the two different positions for SGB (supine with neck rotation and lateral decubitus) may provide data on accuracy, efficacy, and complications, thus facilitating the choice between the two positions. Second, our study was performed on healthy volunteers. The clinical value of our findings needs to be confirmed in further studies on patients who require SGB. Third, intra- and inter-observer analysis of the sonographic assessment of the phrenic nerve was not conducted. Fourth, the values of the angle formed by anterior tubercle, posterior tubercle and phrenic nerve ( $\angle$ CEA) had large variation due to the small number of subjects. Thus, further larger group studies are needed. However, we aimed at not only measuring the angle but also evaluating the change of the value according to the posture. Our results indicated the amount of change and increasing tendency of the angle by changing to lateral decubitus position.

US is a useful tool for localizing the phrenic nerve and assessing its anatomical relations with other structures in the cervical region during US-SGB. The angle formed by anterior tubercle, posterior tubercle and phrenic nerve was significantly wider in the lateral decubitus position than in the supine position, providing larger maneuvering space for the needle. In conclusion, the lateral decubitus position may be a safer technique because it is less likely to result in injury to the phrenic nerve and other related anatomical structures.

\section{CONFLICT OF INTEREST}

No potential conflict of interest relevant to this article was reported.

\section{REFERENCES}

1. Elias M. Cervical sympathetic and stellate ganglion blocks. Pain Physician 2000;3:294-304.

2. Kessler J, Schafhalter-Zoppoth I, Gray AT. An ultrasound study of the phrenic nerve in the posterior cervical triangle: implications for the interscalene brachial plexus block. Reg Anesth Pain Med 2008;33:54550.

3. Canella C, Demondion X, Delebarre A, Moraux A, Cotten H, Cotten A. Anatomical study of phrenic nerve using ultrasound. Eur Radiol 2010;20:659-65.

4. Gofeld M, Bhatia A, Abbas S, Ganapathy S, Johnson M. Development and validation of a new technique for ultrasound-guided stellate ganglion block. Reg Anesth Pain Med 2009;34:475-9.

5. Narouze SN. Ultrasound-guided cervical spine injections: ultrasound "prevents" whereas contrast fluoroscopy "detects" intravascular injections. Reg Anesth Pain Med 2012;37:127-30.

6. Narouze SN, Vydyanathan A, Kapural L, Sessler DI, Mekhail N. Ultrasound-guided cervical selective nerve root block: a fluoroscopy-controlled feasibility study. Reg Anesth Pain Med 2009;34:343-8.

7. Saranteas T, Paraskeuopoulos T, Anagnostopoulou S, Kanellopoulos I, Mastoris M, Kostopanagiotou G. Ultrasound anatomy of the cervical paravertebral space: a preliminary study. Surg Radiol Anat 2010;32:617-22.

8. Siegenthaler A, Mlekusch S, Schliessbach J, Curatolo $M$, Eichenberger U. Ultrasound imaging to estimate risk of esophageal and vascular puncture after conventional stellate ganglion block. Reg Anesth Pain Med 2012;37:224-7.

9. Abdi S, Zhou Y, Patel N, Saini B, Nelson J. A new and 
easy technique to block the stellate ganglion. Pain Physician 2004;7:327-31.

10. Wallace MS, Milholland AV. Contralateral spread of local anesthetic withstellate ganglion block. Reg Anesth 1993;18:55-9.

11. Mak PH, Irwin MG, Ooi CG, Chow BF. Incidence of diaphragmatic paralysis following supraclavicular brachial plexus block and its effect on pulmonary function. Anaesthesia 2001;56:352-6.

12. Robaux S, Bouaziz H, Boisseau N, Raucoules-Aime M, Laxenaire MC; S.O.S. Regional Hot Line Service. Per- sistent phrenic nerve paralysis following interscalene brachial plexus block. Anesthesiology 2001;95:151921.

13. Boon AJ, Alsharif KI, Harper CM, Smith J. Ultrasoundguided needle EMG of the diaphragm: technique description and case report. Muscle Nerve 2008;38:16236.

14. Bhatia A, Flamer D, Peng PW. Evaluation of sonoanatomy relevant to performing stellate ganglion blocks using anterior and lateral simulated approaches: an observational study. Can J Anaesth 2012;59:1040-7. 